\title{
Large-area MEMS-based distributed Bragg reflectors for short-wave and mid-wave infrared hyperspectral imaging applications
}

\author{
Dhirendra Kumar Tripathi, Member, IEEE, Haifeng Mao, K. K. M. B. Dilusha Silva, Member, IEEE, John W. \\ Bumgarner, Mariusz Martyniuk, John M. Dell, Member, IEEE, Lorenzo Faraone, Fellow, IEEE,
}

\begin{abstract}
We present the design, fabrication, and optical characterization, of silicon-air-silicon based distributed Bragg reflectors or quarter wavelength mirrors, in sizes ranging from $200 \mu \mathrm{m}$ $\times 200 \mu \mathrm{m}$ to $5 \mathrm{~mm} \times 5 \mathrm{~mm}$. Such mirrors can be used in conjunction with either single-element photodetectors or large-area focal plane arrays to realise tunable multispectral sensors or adaptive focal plane arrays from the short-wave infrared wavelength ranges $(1500-3000 \mathrm{~nm})$ to mid-wave infrared wavelength $(3000$ $6000 \mathrm{~nm}$ ) ranges. Surface optical profile measurements indicate a flatness of the order of $20-30 \mathrm{~nm}$ in the fabricated structures across several millimetres. Single point spectral measurements on devices show excellent agreement with simulated optical models. The fabricated distributed Bragg reflectors show around 94\% reflectivity, which is in close agreement with theoretical reflectivity. The demonstrated high reflectivity across a wide wavelength range, renders them suitable as broadband reflectors. Finally we present optical transmittance modelling results for Fabry-Pérot filters based on these distributed Bragg reflectors.
\end{abstract}

Keywords-Focal plane array, distributed Bragg reflector, FabryPérot filter, short-wave infrared, mid-wave infrared.

\section{INTRODUCTION}

$\mathbf{T}$ He short-wave infrared (SWIR) wavelength region and mid-wave infrared (MWIR) wavelength region are used extensively in image recognition, detection of trace chemical compounds, security and defense surveillance, and hyperspectral imaging [1]-[6]. MEMS based optical filters and distributed Bragg reflectors (DBRs) have penetrated many applications such as optical spectrometers, digital image projectors and imaging devices [7]-[9]. In this paper we present a silicon-air-silicon (3-layer) DBR or quarter-wave mirror (QWM) for use in MEMS based SWIR and MWIR wavelength range optical devices. We have fabricated DBRs varying in size from $200 \mu \mathrm{m} \times 200 \mu \mathrm{m}$ to $5000 \mu \mathrm{m} \times 5000 \mu \mathrm{m}$. The small size DBRs can be used in applications such as single-pixel MEMS field-portable spectrometers for stand-off detection [10] and the large DBRs can be used in hyperspectral imaging devices to realize large area adaptive focal plane arrays [11]. Fabricating the DBRs ranging in size from $200 \mu \mathrm{m} \times 200 \mu \mathrm{m}$ to several $\mathrm{mm}^{2}$ raises challenges to control the properties of deposited silicon films. Important characteristics of these films include low surface roughness, low tensile stress and good optical

Authors are with the School of Electrical, Electronic and Computer Engineering, University of Western Australia, Perth, WA 6009, Australia. Contact e-mail: dhirendra.tripathi@ research.uwa.edu.au,dilusha.silva@uwa.edu.au. quality. A 3-layer DBR consists of two quarter-wave thick high refractive index dielectric layers separated by a quarter wave thick low refractive index material layer. The higher the contrast between the high and low refractive index, the higher the reflectivity of the DBR.

Taking advantage of mature silicon based technologies, many research groups have used bulk-micromachining of silicon to fabricate high quality in-plane silicon-air-silicon based DBRs in the SWIR wavelength band [12], [13]. These DBRs were small in size and designed for optical communication applications. A comprehensive overview of advances in inplane silicon-air-silicon based DBRs can be found in Ref. [14]. Tuohiniemi et al. [15] fabricated Si-air-Si based DBRs for the long wave infrared (LWIR) wavelength range. In order to avoid stiction associated with wet release of the membranes, they used anti-stiction bumps at the expense of reduced effective optical area. Ebermann et al. [16] used multilayer silicon and silicon oxide structures to fabricate a highly reflecting DBR in the MWIR wavelength range. It was highlighted that use of such multi-materials in a DBR causes stress mismatch in the layers, which must be compensated for in order to achieve a flat surface profile for the DBRs. The same research group also fabricated a dual-band tunable Fabry-Pérot filter for MWIR and LWIR wavelength operation [17]. However, the aperture of the filters and DBRs was limited to $1.9 \mathrm{~mm}$ diameter. Kozak et al. [18] proposed integration of a DBR with a HgCdTe detector to create a Fabry-Perot interferometer centered in the MWIR spectrum. In order to avoid the tendency of warping due to imperfect optical surfaces, they used a SOI-MUMPS process to create the DBRs. With monolithically integrated approaches, one is limited by the choice of materials compatible with both the FPA and the MEMS-based tunable filter fabrication. Furthermore, monolithic integration cannot take full advantage of the myriad of available processes to optimize the stress and optical properties that are available exclusively to MEMS technologies, and the many optimization technologies available exclusively for detector fabrication. In this work we present the fabrication process of large-area silicon-air-silicon DBRs based on thin-film surface micromachining technologies. This is followed by a presentation of the optical measurements and modelling results.

\section{OPTICAL MODELING OF DBRS}

The optical transfer matrix modelling of a multilayer stack of thin films relates the electric and magnetic field at all 
the interfaces of optical thin films via a characteristic matrix. Details of the optical transfer matrix modelling can be found in standard references [19].The optical transfer matrix model has been developed for normal and angled incidence on transparent and weakly absorbing substrates, by assuming that light enters from free space in a thin film with thickness $d_{1}$ and refractive index $n_{1}$ at $\theta_{1}$ angle of incidence. If $\delta$ is the path difference created by the propagation through the thin film, then

$$
\delta=\frac{2 \pi}{\lambda} n_{1} d_{1} \cos \theta_{1} .
$$

The characteristic matrix for the film can be given as

$$
\left[\begin{array}{l}
B \\
C
\end{array}\right]=\left[\begin{array}{cc}
\cos \delta_{1} & \left(\mathrm{i} / \eta_{1}\right) \sin \delta_{1} \\
\mathrm{i} \eta_{1} \sin \delta_{1} & \cos \delta_{1}
\end{array}\right]\left[\begin{array}{c}
1 \\
\eta_{0}
\end{array}\right]
$$

where $\eta_{0}$ is the optical admittance of free space. This result can be extended for a stack of $\mathrm{m}$ thin films, by simply multiplying the characteristic matrix of each film in the order of their appearance.

$$
\left[\begin{array}{l}
B \\
C
\end{array}\right]=\prod_{r=1}^{m}\left(\left[\begin{array}{cc}
\cos \delta_{r} & \left(\mathrm{i} / \eta_{r}\right) \sin \delta_{r} \\
\mathrm{i} \eta_{r} \sin \delta_{r} & \cos \delta_{r}
\end{array}\right]\right)\left[\begin{array}{c}
1 \\
\eta_{\text {out }}
\end{array}\right]
$$

where

$$
\delta_{r}=\frac{2 \pi}{\lambda} n_{r} d_{r} \cos \theta_{r}
$$

The optical admittance can be given as

$$
\begin{gathered}
\eta_{r}=n_{r} \cos \theta_{r}, \text { fors }- \text { polarization }(T E) \\
\eta_{r}=n_{r} / \cos \theta_{r}, \text { forp }- \text { polarization }(T M)
\end{gathered}
$$

where $\eta_{\text {out }}$ is the optical admittance of the output medium. The reflection and transmission coefficients for the dielectric stack are then given by

$$
\begin{aligned}
\rho & =\frac{B \eta_{0}-C}{B \eta_{0}+C} \\
t & =\frac{2 \eta_{0}}{\eta_{0} B+C} .
\end{aligned}
$$

The reflectance of the dielectric stack can be expressed as

$$
R=\left(\frac{B \eta_{0}-C}{B \eta_{0}+C}\right)\left(\frac{B \eta_{0}-C}{B \eta_{0}+C}\right)^{*} .
$$

Garmire [20] calculated that the highest reflectivity for a $\mathrm{N}$ order DBR can be achieved by having the high refractive index layer as the first layer receiving the incident signal from free space. For normal incidence at the central design wavelength $\lambda_{0}$ the reflectivity of a lossless N-period DBR deposited on a substrate with refractive index $n_{S}$ having a high-index first layer is given by

$$
R(N)=\left(\frac{1-n_{S}\left(n_{H} / n_{L}\right)^{2 N}}{1+n_{S}\left(n_{H} / n_{L}\right)^{2 N}}\right)^{2}
$$

and for a $\mathrm{N}+1 / 2$ period $\mathrm{DBR}$, by

$$
R(N+1 / 2)=\left(\frac{n_{S}-n_{H}^{2}\left(n_{H} / n_{L}\right)^{2 N}}{n_{S}+n_{H}^{2}\left(n_{H} / n_{L}\right)^{2 N}}\right)^{2}
$$

Equations 10 and 11 indicate that the reflectivity of a DBR is also dependent on the refractive index of the substrate. This refractive index determines the reflectivity of the interface between the last layer of the mirror and the substrate. The top mirror of a typical MEMS-based Fabry-Pérot filter is usually suspended in air so that $n_{S}=1$, in which case the highest reflectivity is achieved with an $\mathrm{N}+1 / 2$ period mirror, compared to either an $\mathrm{N}+1$ period mirror or an $\mathrm{N}-1$ period mirror. The reflectance finesse of the Fabry-Pérot filter $F_{R}$, is given by

$$
F_{R}=\frac{\pi \sqrt{R}}{1-R} .
$$

The full width at half maximum (FWHM) of a Fabry-Pérot filter $\delta \lambda$, is given by

$$
\delta \lambda=\frac{\lambda}{F_{R}\left(m+\frac{1}{n_{H}-n_{L}}\right)}
$$

where $\mathrm{m}$ is the operating order of the Fabry-Pérot filter. The free spectral range (FSR) can be expressed as

$$
\Delta \lambda=\frac{\lambda}{\left(m+1+\frac{1}{n_{H}-n_{L}}\right)} .
$$

Assuming that $T_{1}$ and $T_{2}$ are the transmittance through the top and bottom DBR of a Fabry-Pérot filter, the transmittance for the Fabry-Pérot filter can be expressed as [21]

$$
\begin{aligned}
& T_{F}(\Psi)=\frac{\frac{T^{2}}{(1-R)^{2}}}{1+\frac{4 F_{R}^{2}}{\pi^{2}} \sin ^{2}\left(\frac{\Psi}{2}\right)} \\
& T_{F}(\Psi)=\frac{T_{\max }}{1+\frac{4 F_{R}^{2}}{\pi^{2}} \sin ^{2}\left(\frac{\Psi}{2}\right)}
\end{aligned}
$$

where $T_{\max }$ is the peak transmittance, $T=\sqrt{T_{1} T_{2}}$, and $\Psi$ represents the round trip phase change. This mathematical model has been used in simulating theoretical models of DBRs and Fabry-Pérot filters.

\section{Structural Design OF DBRs}

An essential consideration for fabricating large area DBRs is the robustness of the support structure under stress caused by the large area suspended membrane. The design used for fabricating large area DBRs is shown in Fig. 1. In this design the suspended membrane has conformal support around the entire periphery, which provides the most robust support structure for a suspended membrane. The fabrication and characterization of the DBRs with this design is presented in this paper. The sacrificial material can be removed through the $5 \mu \mathrm{m}$ diameter etch holes perforated in the top suspended membrane. The adjacent etch holes are $50 \mu \mathrm{m}$ apart, thus, the average porosity of the second suspended silicon layer was reduced by less than $1 \%$. With such a low porosity there is no impact on the refractive index and stress of the suspended silicon layer. In order to block stray light entering through the etch holes, metal pads with $8 \mu \mathrm{m}$ diameter were deposited directly under each etch hole. The presence of the metal pads 
reduces the effective optical area of the DBR by less than $1.5 \%$, which is very minor and has no observed impact on the optical performance of the DBRs.

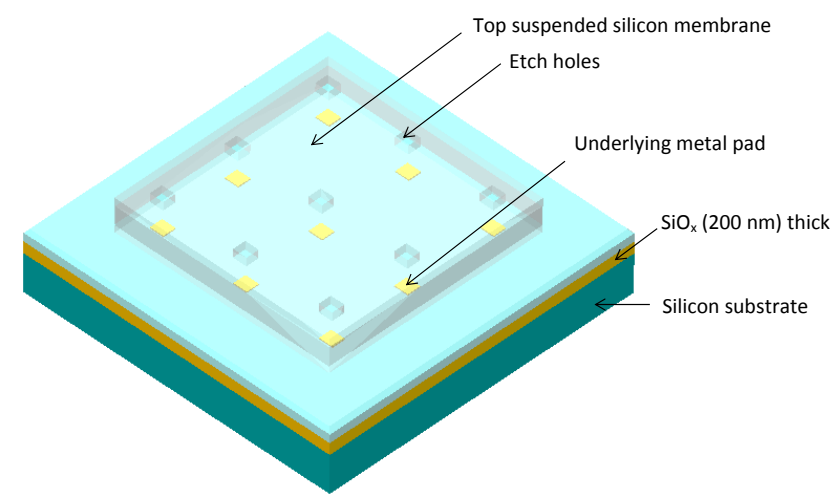

Fig. 1. Structural design of DBRs with conformal support around the entire periphery.

\section{EXPERIMENTAL}

In this work, silicon has been used as the high refractive index material and air has been used as the low refractive index material in the DBRs. The underlying $\mathrm{Si}$ and $\mathrm{SiO}_{\mathrm{x}}$ thin film layers were deposited on $300 \mu \mathrm{m}$ thick $<100>$ oriented silicon substrates using a Sentech SI500D inductively coupled plasma chemical vapour deposition (ICPCVD) system. A Zygo Newview white light optical surface profiler was used to measure surface flatness of the suspended top silicon layers. Table I gives the deposition parameters for ICPCVD $\mathrm{SiO}_{\mathrm{x}}$ films, while Table II gives the deposition parameters for the Si films. In order to measure stress in the Si films, the thin films were deposited on $70 \mu \mathrm{m}$ thick $<100>\mathrm{Si}$ substrates. By measuring stress induced bowing in the thin $\mathrm{Si}$ substrate and applying Stoney's formula [22], [23], the residual stress in deposited Si thin films was calculated, which was found to be dependent on the thickness of the deposited film. This dependency is shown in Fig. 2. A $50 \mathrm{~nm}$ thick silicon film shows $135 \mathrm{MPa}$ of residual compressive stress, which reduces with an increase in the deposited thickness. At $150 \mathrm{~nm}$ thickness the silicon film shows $40 \mathrm{MPa}$ of residual compressive stress which turns into $26 \mathrm{MPa}$ of residual tensile stress for films with $275 \mathrm{~nm}$ thickness. After reaching $90 \mathrm{MPa}$ of residual tensile stress at $500 \mathrm{~nm}$ thickness, the films again show residual compressive stress of $100 \mathrm{MPa}$ for $700 \mathrm{~nm}$ thick films, which reduces to $30 \mathrm{MPa}$ of residual compressive stress at $1000 \mathrm{~nm}$ thickness. The published literature shows similar dependency of stress on the thickness for PECVD oxides [24], [25]. Since the discussion of physical causes of such dependency of stress on thickness is beyond the scope of this paper, we will simply use these values in the fabrication process for DBRs.

\section{FABRICATION PROCESS OF SILICON-AIR-SILICON} BASED LARGE AREA DISTRIBUTED BRAGG REFLECTORS

Fig. 3 shows the fabrication flow of the Si-air-Si DBRs. The fabrication steps are listed as given below:

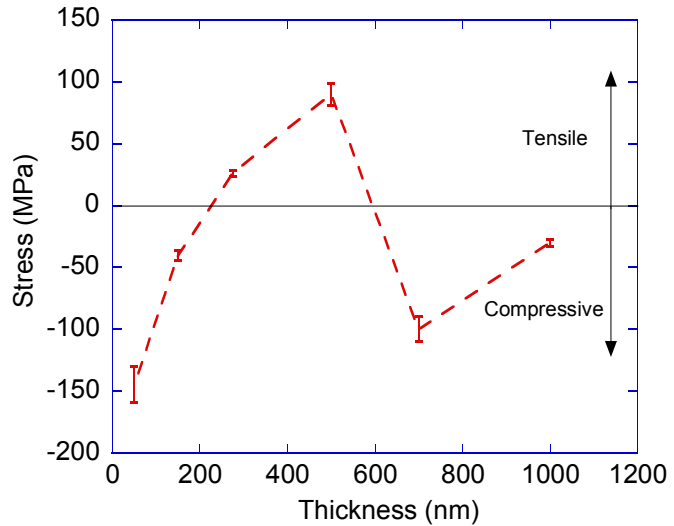

Fig. 2. Stress in ICPCVD deposited silicon thin films as a function of film thickness.

TABLE I. ICPCVD $\mathrm{SiO}_{\mathrm{x}}$ DEPOSITION PARAMETERS.

\begin{tabular}{c||c||c||c||c||c||c}
\hline $\begin{array}{c}\text { ICP } \\
\text { power } \\
(\mathrm{W})\end{array}$ & $\begin{array}{c}\text { Pressure } \\
(\mathrm{Pa})\end{array}$ & $\begin{array}{c}\text { Temperature } \\
\left({ }^{\circ} \mathrm{C}\right)\end{array}$ & $\mathrm{SiH}_{4}$ & $\mathrm{He}$ & $\mathrm{N}_{2} \mathrm{O}$ & $\mathrm{Ar}$ \\
\hline \hline 450 & 2 & 130 & 6.5 & 123 & 70 & 120 \\
\hline
\end{tabular}

TABLE II. ICPCVD DEPOSITION PARAMETERS FOR Si THIN FILMS.

\begin{tabular}{|c|c|c|c|c|}
\hline \multirow{2}{*}{$\begin{array}{l}\text { ICP power } \\
\text { (W) }\end{array}$} & \multirow{2}{*}{$\begin{array}{c}\text { Pressure } \\
(\mathrm{Pa})\end{array}$} & \multirow{2}{*}{$\begin{array}{c}\text { Temperature } \\
\left({ }^{\circ} \mathrm{C}\right)\end{array}$} & \multicolumn{2}{|c|}{ Flow rate $(\mathrm{sccm})$} \\
\hline & & & $\mathrm{SiH}_{4}$ & $\mathrm{He}$ \\
\hline 26 & 4 & 300 & 5 & 95 \\
\hline
\end{tabular}

TABLE III. ACTUAL THICKNESS OF DEPOSITED LAYERS FOR MWIR AND SWIR DBRS.

\begin{tabular}{c||c||c}
\hline Layer & $\begin{array}{c}\text { Thickness (MWIR DBR) } \\
(\mathrm{nm})\end{array}$ & $\begin{array}{c}\text { Thickness (SWIR DBR) } \\
(\mathrm{nm})\end{array}$ \\
\hline \hline & & \\
$\mathrm{SiO}_{\mathrm{x}}$ & 200 & 200 \\
$\mathrm{Si}[1]$ & 345 & 158 \\
Prolift100-16 & $1230-1300$ & $500-520$ \\
$\mathrm{Si}[2]$ & 335 & 145 \\
\hline
\end{tabular}

1) As shown in Fig. 3(a) we start with a silicon substrate and deposit a $200 \mathrm{~nm}$ silicon oxide layer. This thickness was chosen simply for convenience, and was the same for both MWIR and SWIR devices. For an optimized DBR this layer should be one quarter wavelength thick. On top of the oxide layer a quarter wavelength thick silicon layer is deposited, which is the first optical layer of the DBR. For the MWIR and SWIR DBRs the target quarter wavelength thickness of silicon thin films was $350 \mathrm{~nm}$ and $145 \mathrm{~nm}$, respectively. The actual deposited thickness of the deposited films was different from these values, and Table III shows the actual thickness of the deposited layers.

2) On top of the first silicon layer a chromium $(10 \mathrm{~nm}) /$ gold $(100 \mathrm{~nm})$ metal bottom optical shield is deposited which also deposits optical signal blocking metal pads underneath the etch holes (see Fig. 3 (b)). 


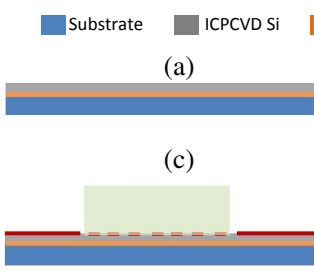

(e)

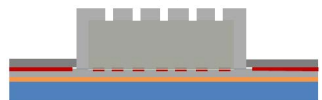

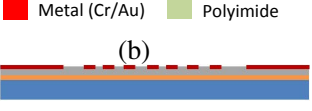

(d)

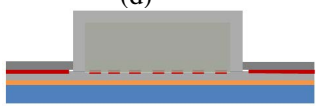

(f)

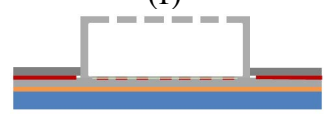

Fig. 3. Fabrication process of DBRs. (a) Deposition of quarter wavelength thick Si thin film on silicon oxide deposited substrate; (b) deposition of metal optical shield; (c) spinning, hard-baking and patterning of PL100-16 polyimide ; (d) deposition of the second quarter wavelength thick Si thin film on top of polyimide; (e) patterning of etch holes through the top Si membrane; (f) dry release of DBR in the $\mathrm{O}_{2}$ plasma.

3) Brewer science Prolift 100-16 was chosen as the sacrificial layer. Apex-k1-100 adhesion promoter was spun at $4000 \mathrm{rpm}$ and baked at $150{ }^{\circ} \mathrm{C}$ for 30 seconds. In order to achieve a quarter wavelength thick air cavity, the Prolift100-16 was spun at $1800 \mathrm{rpm}$ for MWIR DBRs and, at $4000 \mathrm{rpm}$ for SWIR DBRs. The sacrificial layer was soft-baked at $100{ }^{\circ} \mathrm{C}$ for 2 minutes and then ramped to $250{ }^{\circ} \mathrm{C}$ for a total of 15 minutes to fully cure it. The sacrificial layer was wet patterned in AZ326 developer with a resulting thickness as shown in Table III (Fig. 3(c)).

4) After the removal of the photoresist a short oxygen plasma exposure was performed for 15 seconds, which improves adhesion of the top silicon layer to the sacrificial layer. On top of the sacrificial layer a second silicon layer was deposited, which conformally covers the sacrificial layer (Fig. 3(d)).

5) The top silicon layer was perforated with $5 \mu \mathrm{m}$ diameter etch holes using $\mathrm{CF}_{4}$ plasma as shown in Fig. 3 (e). As shown in Fig. 2, silicon thin films with thickness around $150 \mathrm{~nm}$ will be compressive in nature, hence for SWIR DBRs a pre-release annealing at $260{ }^{\circ} \mathrm{C}$ for 30 minutes was performed in a quartz tube furnace in nitrogen ambient. The silicon thin films with thickness around $350 \mathrm{~nm}$ were tensile in nature, hence MWIR DBRs were not annealed. The dependence of residual stress on thin film thickness has been extensively reported by several authors [26], [27]. It was observed that the stress in thin films was initially compressive in nature, and that with increasing in thickness enters into a tensile region. A further increase in film thickness eventually renders it increasingly compressively stressed. Furthermore, Leib et al. [28], [29] explored the possibility of a reversible change in stress in the compressive stress region. Our findings of dependence of stress of silicon thin films on deposited thickness of silicon thin films, shown in Fig. 2, are in agreement with these previous studies.

6) The sacrificial layer was etched in an $\mathrm{O}_{2}$ plasma in a barrel Asher with $160 \mathrm{~W}$ RF power and $133.3 \mathrm{~Pa}$ chamber pressure. The removal of the sacrificial layer leaves the top membrane suspended over the bottom layer, thus forming an air cavity as shown in Fig. 3 (f).

DBRs ranging in size from $200 \mu \mathrm{m} \times 200 \mu \mathrm{m}$ to $5 \mathrm{~mm}$ $\times 5 \mathrm{~mm}$ were fabricated using the above fabrication process. A microscope image of a $2 \mathrm{~mm} \times 2 \mathrm{~mm}$ fabricated DBR is shown in Fig. 4.

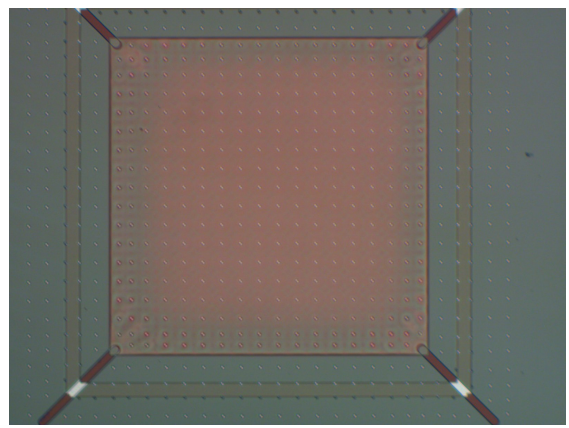

Fig. 4. Micrograph of a $2 \mathrm{~mm} \times 2 \mathrm{~mm}$ fabricated DBR.

\section{OPTICAL CHARACTERISATION OF DBRS}

The effectiveness of the DBR fabrication process was assessed through optical characterization. Optical surface profilometry and optical transmittance measurements were chosen to optically characterize the DBRs.

\section{A. Optical surface profilometery}

A line scan across a $2 \mathrm{~mm} \times 2 \mathrm{~mm}$ DBR for SWIR wavelengths is shown in Fig. 5 (a), and its expanded scale plot is shown Fig. 5 (b). Note the variation of the flatness in the center $1.45 \mathrm{~mm}$ length across the $2 \mathrm{~mm}$ mirror was found to be in the range of 10-20 nm. In this DBR the edges shows a sharp bowing of 40-50 $\mathrm{nm}$. An expanded scale plot of the surface profile indicates the available optical area for the SWIR DBR, indicating a very low flatness variation of the order of 10-20 nm.

All fabricated MWIR and SWIR wavelength range DBRs, of all sizes, show sharp bowing at the edges, and have 70$75 \%$ of the fabricated area being optically flat to within a 20$30 \mathrm{~nm}$ range. This flat area can be designated as the optically active area because the variation in flatness within a $20-30 \mathrm{~nm}$ range will cause a very insignificant change in the optical performance of the DBRs. The sharp bowing at the edges of each DBR is most likely due to the anchor effect resulting from tensile stress in the silicon thin films. This bowing can be reduced by reducing the tensile stress from the present value to further lower values. The variation in bowing at the edge of the DBRs with variation in the stress in amorphous silicon thin films will be the subject of future study. 
(a)

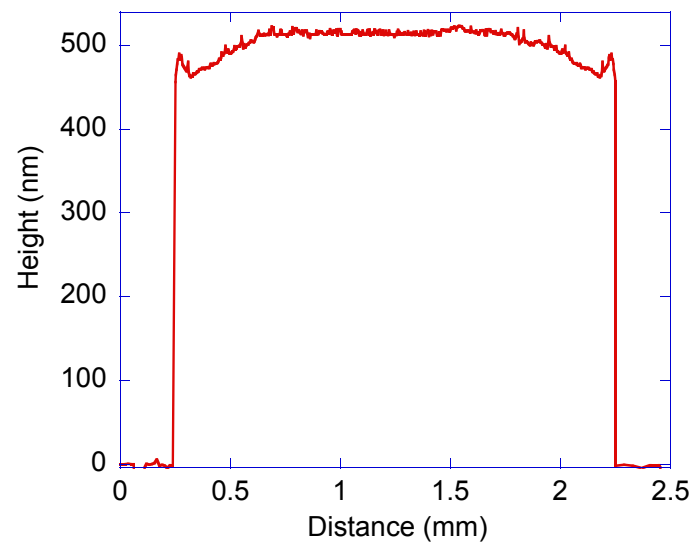

(b)

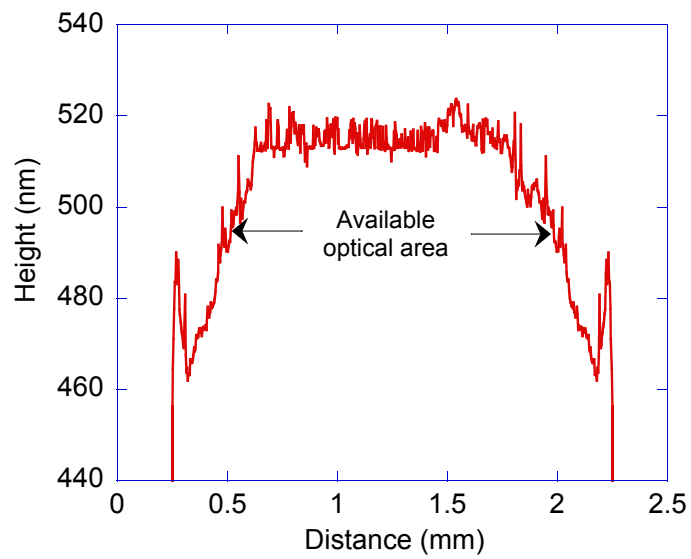

Fig. 5. (a) A line scan across surface profile of $2 \mathrm{~mm} \times 2 \mathrm{~mm}$ DBR in the SWIR wavelength range avoiding etch holes; (b) an expanded scale surface profile of (a).

\section{$B$. Single point transmittance and reflectance spectrum of the fabricated DBRs}

The optical transmission of DBRs was investigated using a Spectrum One FTIR spectrometer by Perkin Elmer. This system enables measurement of spectral transmission and absorption in the spectral range from $1.3 \mu \mathrm{m}$ to $28.5 \mu \mathrm{m}$ with high accuracy. The beam spot size for optical measurement of DBRs was $\geq 2 \mathrm{~mm} \times 2 \mathrm{~mm}$ in size which is much larger than the periodicity and size of the etch holes and metal pad patterns in the fabricated DBRs. Due to the large beam spot size, and the low density and small size of etch holes and metal pad patterns, we expect scattering effects on the final optical measurements to be negligible. However, with our current instrument setup and accessories, a specular reflection measurement was not possible. Hence a transmission measurement was used to estimate the reflectance of the DBRs.

Figure 6 shows the measured single point transmission (optical beam diameter of $1 \mathrm{~mm}$ ), and the simulated and estimated reflectance for a $2 \mathrm{~mm} \times 2 \mathrm{~mm}$ DBR was $1 \mathrm{~mm}$ in diameter. The simulated spectra was obtained by the optical transfer matrix method described in Section II. The simulated models used actual measured thicknesses and optical constants of ICPCVD silicon and silicon oxide layers. However, at this point the simulated models do not include the effect of surface variations. The extracted extinction coefficient of amorphous silicon thin films was negligible (in the order of $10^{-6}$ ) in the SWIR and MWIR wavelength range, which indicates that the DBRs have negligible absorption. Hence, the percentage reflectance can readily be calculated from the measured percentage transmittance by direct subtraction from $100 \%$. The "Calculated Reflectance" shows the final calculated reflectivity of fabricated DBRs.

Figure 6 (a) and (b) shows the optical spectrum of MWIR and SWIR DBRs, respectively. As shown in Fig. 6 (a) the measured minimum transmission of $5 \%$ compares well with the $4.5 \%$ predicted from the simulation. In comparison to the theoretical $94.5 \%$ reflectivity, we attained $94 \%$ peak reflectivity. The DBRs show more than $85 \%$ reflectivity over the $3750 \mathrm{~nm}-6000 \mathrm{~nm}$ wavelength range. The reflectivity is close to $93 \%$ in the wavelength range $4100-4910 \mathrm{~nm}$. Figure 6 (b) shows that $5.7 \%$ is the minimum measured transmittance of SWIR DBRs, which compares well with the theoretical $5.2 \%$ predicted for the simulated model. The SWIR DBR shows $94.3 \%$ reflectivity as compared to $94.8 \%$ simulated reflectivity. The SWIR DBRs show more than $85 \%$ reflectivity over the $1950 \mathrm{~nm}-2700 \mathrm{~nm}$ wavelength range. Thus, the very flat surface profile variation and high reflectivity over a wide wavelength range can be effectively utilized in a range of spectroscopic imaging systems. It is to be noted that the fabricated DBR can deliver the highest performance by choosing a quarter wavelength thick silicon oxide layer on the substrate. Figure 6 (a) and (b) show "Simulated Reflectance (QW SiOx)", which is the simulated reflectivity of DBRs with a quarter wavelength thick silicon oxide layer. For the MWIR wavelength range DBRs, the quarter wavelength thickness of silicon oxide layer is $868 \mathrm{~nm}$. Figure 6 (a) shows that the peak reflectivity for the MWIR wavelength range DBRs increases to $97 \%$, which gives an improvement of $3 \%$ for the peak reflectivity. The DBR shows more than $85 \%$ reflectivity over the $3540 \mathrm{~nm}-$ $7510 \mathrm{~nm}$ wavelength range, which is an increase of $1720 \mathrm{~nm}$ as compared to the fabricated MWIR wavelength range DBRs. In the same way, by choosing a $347 \mathrm{~nm}$ thick oxide layer for the SWIR DBR, the peak reflectivity can be increased to $97 \%$ and more than $85 \%$ reflectivity can be obtained over the $1920 \mathrm{~nm}-3390 \mathrm{~nm}$ wavelength range. Hence, an optimized Si-air-Si based DBR should have a quarter wavelength thick underlying silicon oxide layer in order to achieve maximum reflectivity and widest bandwidth. Fabrication of such DBRs will be included as part of our future work of fabricating FabryPérot filters based on Si-air-Si DBRs.

\section{Fabry-Pérot filter based on Silicon-air-silicon DBRs}

A schematic layout for the proposed Fabry-Pérot filter based on Si-air-Si DBRs is shown in Fig. 7(a), and a cross section is shown in Fig. 7(b). The filter layout is applicable to filters of size $200 \mu \mathrm{m} \times 200 \mu \mathrm{m}$, to $1000 \mu \mathrm{m} \times 1000 \mu \mathrm{m}$. With some modification to the size of the electrodes and notches, this structure can be adapted for even larger filter sizes. The 
(a)

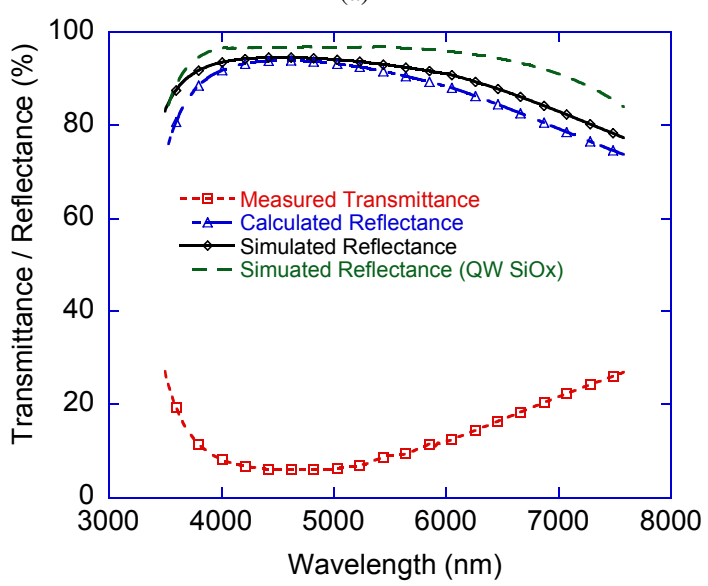

(b)

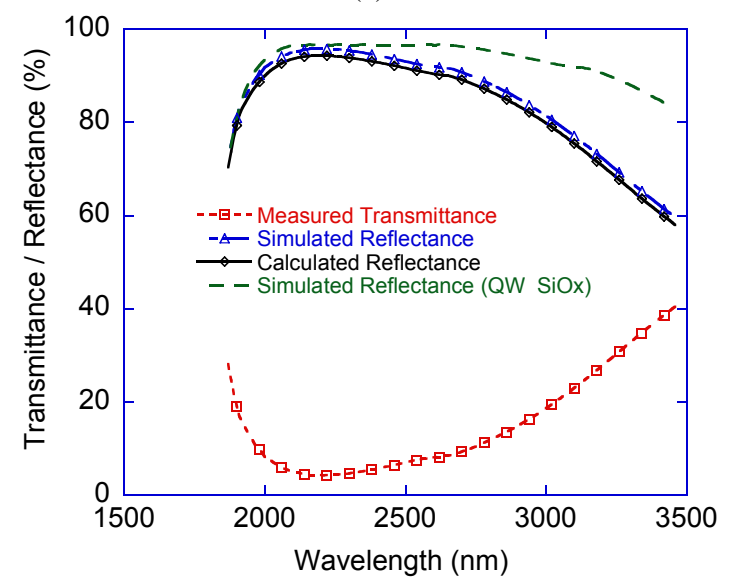

Fig. 6. The transmittance and reflectance of the fabricated DBRs. Measured Transmittance- measured transmittance data; Calculated ReflectanceDBR reflectance based on fitted transmission. Simulated Reflectance-expected reflectance of DBR; Simulated Reflectance (QW SiOx)- expected reflectance of DBR by choosing quarter wavelength thick silicon oxide layer; (a)Transmittance and reflectance values for the MWIR DBRs (b) transmittance and reflectance of SWIR DBRs shown in Fig. 5.

top mirror and bottom mirror are made of Si-air-Si DBRs, with each silicon layer in this structure being quarter wavelength thick. An optical area $10 \mu \mathrm{m}$ smaller than the mirror dimension is opened through the optical signal blocking metal shield deposited on the backside of the substrate. The four top electrodes and four bottom electrodes provide symmetric actuation movement to the top DBR. A specific feature of the top membrane are the notches at the four corners of the top DBR which allow the top membrane to actuate without causing stress induced distortion in the top DBR. The sacrificial layer is etched through the etch holes. Unwanted optical signal through the etch holes is blocked with the help of metallic optical blocking pads underneath each etch hole.

In order to confirm the suitability of the DBRs for tunable Fabry-Pérot filters for hyperspectral imaging applications, we modelled the optical transmission of the filter structure shown in Fig. 7 using the optical transfer matrix model discussed in (a)

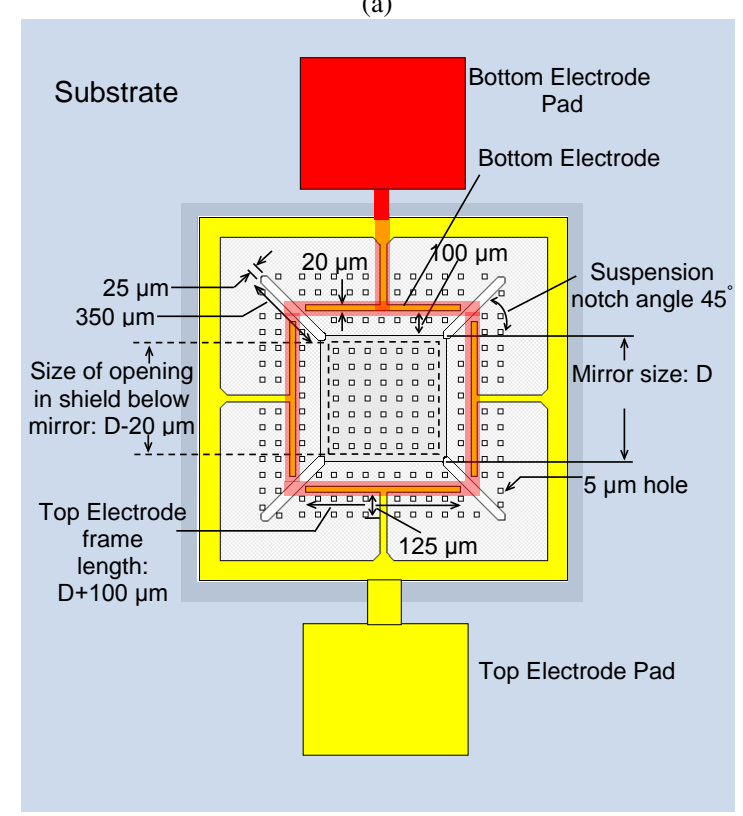

(b)

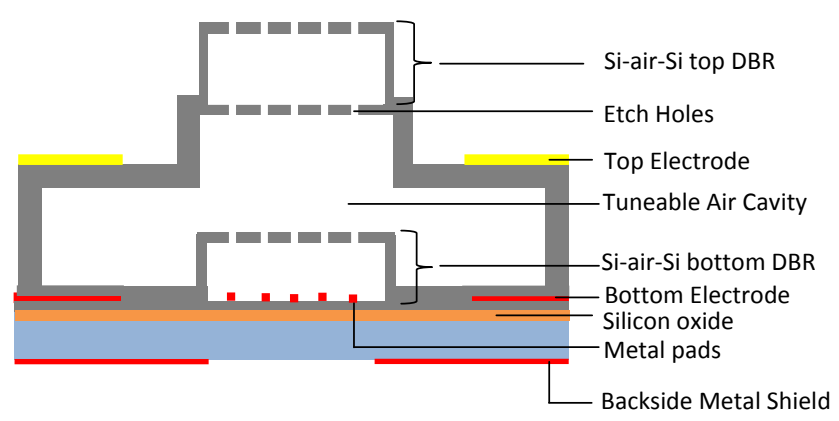

Fig. 7. Graphical layout for the proposed Fabry-Pérot filter. (a) Shows a top view; (b) shows cross section through filter.

Section II. A $310 \mu \mathrm{m}$ thick silicon substrate was used as the substrate in the model. Next, a silicon oxide layer of optimum thickness is placed on top of the substrate. For the SWIR and MWIR wavelength range region the quarter wave thickness of silicon oxide layer was chosen as $470 \mathrm{~nm}$ and $1035 \mathrm{~nm}$, respectively. Both the bottom and top DBRs consisted of siliconair-silicon mirrors, separated by a tunable air cavity gap. For the SWIR and MWIR wavelength range DBRs, the quarter wave thickness of the silicon layers was chosen as $197 \mathrm{~nm}$ and $440 \mathrm{~nm}$, respectively, and the quarter wavelength thick air gap within the DBRs was chosen as $675 \mathrm{~nm}$ and $1500 \mathrm{~nm}$, respectively. The initial length of the tunable air cavity for the MWIR wavelength range and SWIR wavelength range operation was chosen as $3000 \mathrm{~nm}$ and $1350 \mathrm{~nm}$, respectively, which allows the Fabry-Pérot filters to operate over the entire high reflectivity region of the DBRs. The cavity length of the filters was tuned to obtain the transmittance spectrum. Figure 8 (a) and (b) shows modelling results for the MWIR and SWIR filters, respectively. As shown in Fig. 8 (a) the modelling 
predicts that a MWIR wavelength range filter based on Si-airSi DBRs will have a peak transmission $>80 \%$, a minimum $30 \mathrm{~nm}$ FWHM, and a maximum $1850 \mathrm{~nm}$ FSR, thus resulting in a finesse of 61.7 and a quality factor of 200. Fig. 8 (b) shows that a SWIR filter is expected to have a peak transmission $>$ 60\%, a minimum $15 \mathrm{~nm}$ FWHM, a maximum $850 \mathrm{~nm}$ FSR, a finesse of 70, and a quality factor of 180 . It can be seen from Fig. 8 that as the filter is tuned to the shorter wavelength range of the DBRs, the FWHM widens and the out of band rejection ratio reduces significantly due to the lower mirror reflectivity at these shorter wavelengths. This modelling suggests that high finesse and broad FSR filters can be readily realized using Siair-Si based DBRs. Table IV shows a summary of the optical results for the fabricated DBRs and modelled filters. In this table $\Delta \lambda$ indicates the maximum shift in the small-area spot scan peak transmittance across the active optical area of a filter due to the experimentally measured flatness variation across the fabricated DBRs.

\section{CONCLUSION}

In this paper we have presented the design and fabrication process of silicon-air-silicon based distributed Bragg reflectors, operating in the SWIR and MWIR wavelength ranges, and ranging in sizes from $200 \mu \mathrm{m} \times 200 \mu \mathrm{m}$ to $5 \mathrm{~mm} \times 5 \mathrm{~mm}$. The variability of sizes allows them to be used in conjunction with either single-element photodetectors or large-area focal plane arrays. The fabricated DBRs demonstrate a $20-30 \mathrm{~nm}$ variation in flatness across several millimetres in dimension. Single point spectral measurements on devices show excellent agreement with simulated optical models. The fabricated distributed Bragg reflectors have around $94 \%$ reflectivity compared to the theoretical 95-96\% reflectivity. Modelling of Fabry-Pérot filters based on these distributed Bragg reflectors indicated high finesse, narrow FWHM, and wide FSR for both the SWIR and MWIR wavelength ranges.

\section{ACKNOWLEDGMENT}

This work was performed in part at the Western Australian node of the Australian National Fabrication Facility (ANFF). This research was supported financially by the Grains Research and Development Corporation, as well as by a number Australian Research Council research grants (DP 0881579, DP 140101766). We also acknowledge support from the Western Australian State Government Office of Science.

\section{REFERENCES}

[1] M. J. Cantelia, "Space surveillance with medium-wave infrared sensors," The Lincoln Laboratory Journal, vol. 1, no. 1, pp. 75-88, 1988.

[2] E. Ochoa, T. Nelson, R. Bedford, J. Ehret, L. Starman, M. Harvey, T. Anderson, and F. Ren, "Demonstration of hybrid $\mathrm{Al}_{\mathrm{x}} \mathrm{Ga}_{(1-\mathrm{x})} \mathrm{As}$ Polysilicon microelectromechanical tunable filter," IEEE Photonics Technology Letters, vol. 19, no. 6, pp. 381-383, 2007.

[3] M. K. Griffin, Hsiao-Hua, K. Burke, and J. P. Kerekes, "Understanding radiative transfer in the midwave infrared, a precursor to full spectrum atmospheric compensation," in Proceedings of SPIE, vol. 5425, 2004, pp. $348-356$. (a)

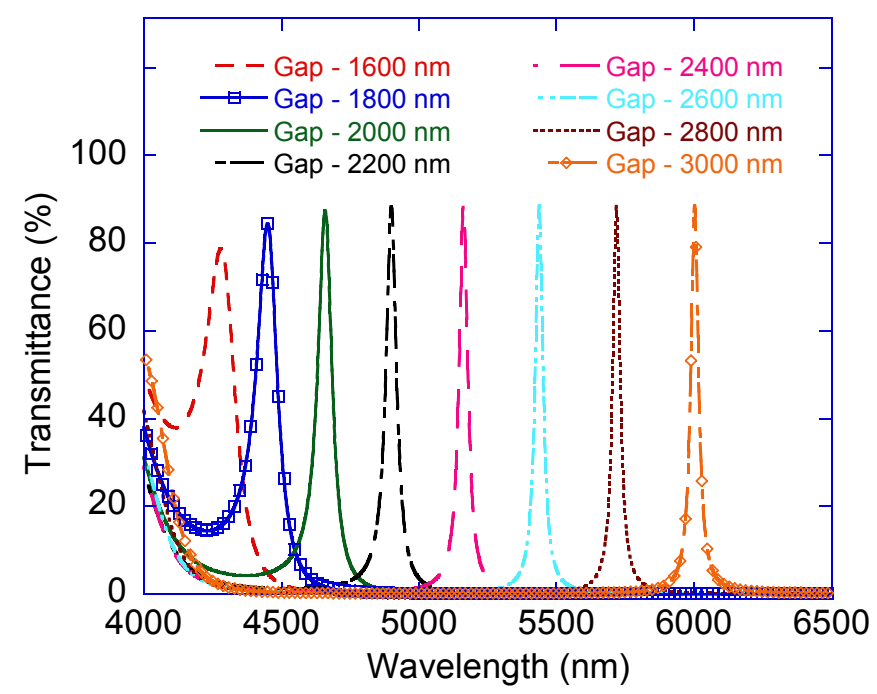

(b)

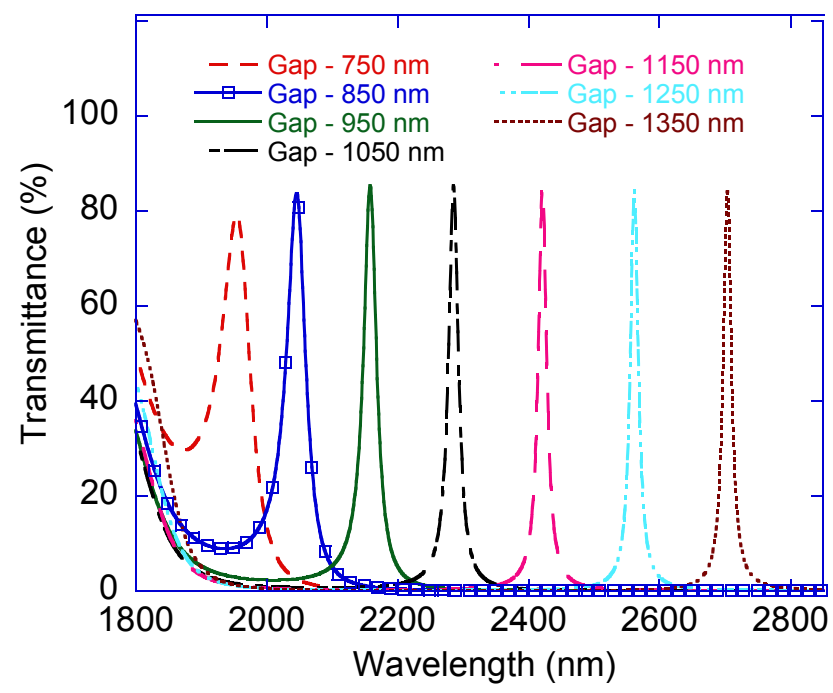

Fig. 8. The modelled transmittance of Fabry-Pérot filters based on Si-air-Si DBRs. (a) Shows transmittance of MWIR filter; (b) shows transmittance of SWIR filter.

[4] L. Rousset-Rouviere, C. Coudrain, S. Fabre, , I. Baarstad, A. Fridman, T. Loke, S. Blaaberg, and T. Skauli, "Sysiphe, an airborne hyperspectral imaging system for the VNIR-SWIR-MWIR-LWIR region," in Proceedings of $7^{\text {th }}$ EARSeL Workshop on Imaging Spectroscopy, vol. 5, 2004, pp. $1-12$.

[5] F. Felder, M. Arnold, M. Rahim, C. Ebneter, and H. Zogg, "Tunable lead-chalcogenide on Si resonant cavity enhanced midinfrared detector," Applied Physics Letters, vol. 91, no. 10, p. 101102, 2007.

[6] N. Osia and T. Bourlai, "A spectral independent approach for physiological and geometric based face recognition in the visible, middle-wave and long-wave infrared bands," Image and Vision Computing, vol. 32, no. 11, pp. $847-859,2014$.

[7] J. Dell, A. Keating, J. S. Milne, J. Antoszewski, C. Musca, L. Faraone, D. Murphy, and O. Samardzic, "Micro-electromechanical systems-based microspectrometers covering wavelengths from $1500 \mathrm{~nm}$ to $5000 \mathrm{~nm}$," in SPIE Defense and Security, Florida, USA, 2007. 
TABLE IV. SUMmary OF OPTICAL RESUlts OF FABRICATED DBRS AND MODELLED FABRY-PÉROT FILTERS.

\begin{tabular}{|c|c|c|c|c|c|c|c|}
\hline & \multicolumn{3}{|c|}{ DBR or Quarter-wave mirror } & \multicolumn{4}{|c|}{ Modelled Filter } \\
\hline Wavelength Range & $\begin{array}{c}\text { Flatness variation of } \\
\text { fabricated DBRs }(\mathrm{nm})\end{array}$ & $\begin{array}{l}\text { Active optical area of } \\
(\mathrm{nm})\end{array}$ & $\begin{array}{l}\text { Wavelength range for } \\
2 \mathrm{~mm} \times 2 \mathrm{~mm} \text { DBR }\end{array}$ & $\begin{array}{c}\text { FWHM } \\
\text { Reflectivity }>85 \%\end{array}$ & $\begin{array}{l}\text { FSR } \\
(\mathrm{nm})\end{array}$ & $\begin{array}{c}\text { Peak Transmittance } \\
(\mathrm{nm})\end{array}$ & $\begin{array}{l}\Delta \lambda \\
(\%)\end{array}$ \\
\hline SWIR $(1500 \mathrm{~nm}-3000 \mathrm{~nm})$ & \pm 20 & $1.45 \mathrm{~mm} \times 1.45 \mathrm{~mm}$ & $1950 \mathrm{~nm}-2700 \mathrm{~nm}$ & 15 & 850 & $>60 \%$ & \pm 30 \\
\hline MWIR (3000 nm-6000 nm) & \pm 30 & $1.55 \mathrm{~mm} \times 1.55 \mathrm{~mm}$ & $3750 \mathrm{~nm}-6000 \mathrm{~nm}$ & 30 & 1850 & $>80 \%$ & \pm 15 \\
\hline
\end{tabular}

[8] R. A. Crocombe, "MEMS technology moves process spectroscopy into a new dimension," Spectroscopy Europe, vol. 16, no. 3, pp. 16-19, 2000.

[9] P. F. V. Kessel, L. J. Hornbeck, R. E. Meier, and M. R. Douglass, "A MEMS-based projection display," in Proceedings of the IEEE, vol. 86, 1998, pp. 1686-1704.

[10] J. S. Milne, J. M. Dell, A. J. Keating, and L. Faraone, "Widely tunable MEMS-based FabryPerot filter," Journal of Microelectromechanical Systems, vol. 18, no. 4, pp. 905-913, 2009.

[11] P. J. Treado, I. W. Levin, and E. N. Lewis, "Indium antimonide (InSb) focal plane array (FPA) detection for near-infrared imaging microscopy," Applied Spectroscopy, vol. 18, pp. 607-615, 1994.

[12] A. Lipson and E. M. Yeatman, "A 1-D photonic band gap tunable optical filter in $<110\rangle$ silicon," Journal of micromechanical systems, vol. 16, no. 3, pp. 521-527, 2007.

[13] B. Saadany, M. Malak, F. Marty, Y. Mita, D. Khalil, and T. Bourouina, "Electrostatically-tuned optical filter based on silicon bragg reflectors," in IEEE/LEOS international conference on optical MEMS and their applications, 2006, pp. 86-87.

[14] R. St-Gelais, A. Poulin, and Y. A. Peter, "Advances in modeling, design, and fabrication of deep-etched multilayer resonators," Journal of Lightwave Technology, vol. 30, no. 12, pp. 1900-1908, 2012.

[15] M. Tuohiniemi and M. Blomberg, "Surface-micromachined silicon airgap bragg reflector for thermal infrared," Journal of Micromechanics and Microengineering, vol. 21, no. 7, pp. 075014[1-7], 2011.

[16] M. Ebermann, K. Hiller, S. Kurth, and N. Neumann, "Design, operation and performance of a Fabry-Perot-Based MWIR microspectrometer," in Proceedings-OPTO 2009 and IRS 2 2009, 2009, pp. 233-238.

[17] M. Meinig, M. Ebermann, N. Neumann, S. Kurth, K. Hiller, and T. Gessner, "Dual-band MEMS FABRY-PEROT filter with two movable reflectors for mid- and long-wave infrared microspectrometers," in 16th International Solid-State Sensors, Actuators and Microsystems Conference (TRANSDUCERS), 2011, pp. 2538 - 2541.

[18] D. A. Kozak, B. Fernandez, M. L. Morley, S. Velicu, and J. Kubby, "Fabrication and testing of a MEMS-based optical filter combined with a HgCdTe detector," in Proc. SPIE MOEMS and Miniaturized Systems $X$, vol. 7930, 2010.

[19] H. A. Macleod, Thin-Film Optical Filters. Institute of Physics, London, 2001.

[20] E. Garmire, "Theory of quarter-wave-stack dielectric mirrors used in a thin Fabry-Perot filter," Appl. Opt., vol. 42, no. 27, pp. 5442-5449, Sep 2003.

[21] J. M. Vaughan, The Fabry-Perot Interferometer History, Theory,Practice and Applications. Taylor and Francis, New York, 1989.

[22] G. G. Stoney, "The tension of metallic films deposited by electrolysis," Proceedings of Royal Society, vol. 82, pp. 172-175, 1909.

[23] X. Feng, Y. Huang, and A. J. Rosakis, "On the Stoney's formula for a thin film/substrate system with non-uniform substrate thickness," Journal of Applied Mechanics, vol. 74, pp. 1276-81, 2007.

[24] V. Au, C. Charles, D. A. P. Bulla, J. D. Love, and R. W. Boswell, "Thickness-dependent stress in plasma-deposited silicon dioxide films," Journal of Applied Physics, vol. 97, no. 8, pp. 84912[1-7], 2005.

[25] R. Lathlaen and D. A. Diehl, "Stress in thin films of silane vapordeposited silicon dioxide," Journal of Electrochemical society, vol. 116, no. 5, pp. 620-622, 1969.

[26] A. L. Shull and F. Spaepen, "Measurements of stress during vapor deposition of copper and silver thin films and multilayers," Journal of Applied Physics, vol. 80, no. 11, pp. 6243-6256, 1996.
[27] F. Spaepen, "Interfaces and stresses in thin films," Acta Materilia, vol. 48, pp. 32-41, 2000.

[28] J. Leib, R. Mönig, and C. V. Thompson, "Direct evidence for effects of grain structure on reversible compressive deposition stresses in polycrystalline gold films," Phys. Rev. Lett., vol. 102, p. 256101, Jun 2009.

[29] J. Leib and C. V. Thompson, "Weak temperature dependence of stress relaxation in as-deposited polycrystalline gold films," Phys. Rev. B, vol. 82, p. 121402, Sep 2010.

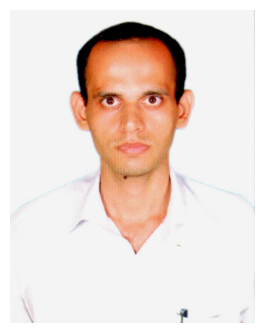

Dhirendra Kumar Tripathi received the B.Tech. degree in electronics engineering from UPTU University, Lucknow, and M.Tech. degree in VLSI Systems from NIT, Trichy, India in 2005 and 2008, respectively. He is currently pursuing the Ph.D. degree in the School of Electrical, Electronic and Computer Engineering in The University of Western Australia, Crawley Perth, Australia. His current research activities involve design and fabrication of optical MEMS devices and materials for the MEMS.

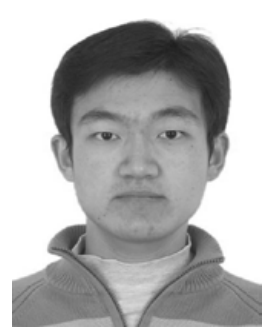

Haifeng Mao was born in China in 1985. He received the B.Eng. degree in Physics from Dalian University of Technology, China, in 2007, and M.Sc. degree in Sensor Systems Technology from Karlsruhe University of Applied Sciences, Germany, in 2010. He is currently pursuing the Ph.D. degree at the School of Electrical, Electronic and Computer Engineering, University of Western Australia, Perth, Australia. His current research activities involve design, fabrication and characterization of optical MEMS filters for multispectral imaging applications.

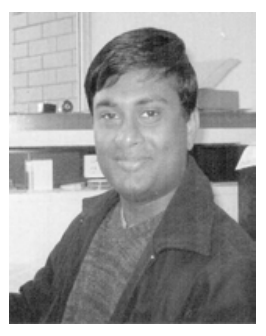

K. K. M. B. Dilusha Silva was born in Sri Lanka in 1973. He received the Honours degrees in Physics and Electronic Engineering from the University of Western Australia (UWA), and the Ph.D. degree in optical imaging technologies for biomedical applications in 2004.

He has worked both in industry and academia, and is currently a Research Professor and the Engineering Manager with the Microelectronics Research Group, UWA. Since returning to UWA in 2009, his research interests include optical MEMS sensors, optical spectroscopic sensors, and MEMS biosensors. He has attracted funding for his research from the agriculture and aerospace sectors, and the government, and is presently leading a number of MEMS related research efforts at MRG, with strong commercial links to both the agricultural and aerospace sectors. 


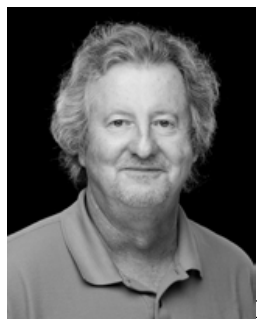

John Bumgarner received the B.S. and M.S. degrees in materials science and engineering from Northwestern University, and the Ph.D. degree in materials science and engineering from North Carolina State University with a focus on wide band gap materials, SiC, and CVD of dielectrics in 1995.

He was a Process Integration Engineer and the Manager with Intel Corporation, a Laboratory Director with SRI International, and the Operations Director with Stanford University NanoFab. He is currently an Associate Research Professor with the University of Western Australia. His current interests are in research and development of optical microelectromechanical systems devices.

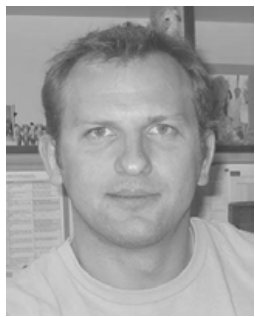

Mariusz Martyniuk was born in Poland in 1976 $\mathrm{He}$ received the B.Sc. (Hons.) degree from the University of Toronto, ON, Canada, the M.A.Sc. degree from McMaster University, ON, Canada, and the Ph.D. degree from the University of Western Australia, Perth, Australia, in 2007.

$\mathrm{He}$ worked in the industry sector as an Electronics Engineer before rejoining UWA, where he is currently a Research Professor with the Microelectronics Research Group and manages the Western Australian Node of the Australian National Fabrication Facility. His primary areas of interest encompass thin-film materials and thin-film mechanics, as well as their applications in micro-electromechanical systems and optoelectronic devices.

Dr. Martyniuks research contributions were recognized by the award of the Inaugural Australian Museum Eureka Prize (the Oscars of Australian science) for Outstanding Science in Support of Defence or National Security in 2008.

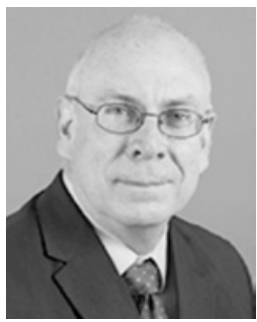

John M. Dell is currently a Professor of Electrical Engineering with the University of Western Australia, Perth, WA, Australia.

His primary areas of interest are semiconductor optoelectronics and optical microelectromechanical systems (MEMS) devices. He has worked in both industry and academia in these fields. Work undertaken by his group on robust and low-cost microspectrometer technology has attracted funding from the US and the Australian Department of Defence, and more recently from the Australian Grains Research and Development Corporation. This latter funding is for the development of lowcost tools using infrared spectroscopy for broad acre agriculture applications. This work is being undertaken as a collaboration between Electrical Engineers and Soil Scientists. He has several patents, and over 200 journal and conference publications

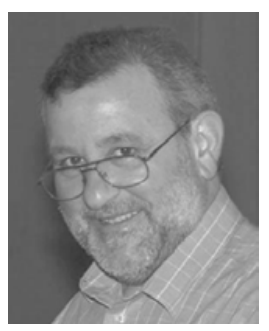

Lorenzo Faraone was born in Italy in 1951. He received the $\mathrm{Ph} . \mathrm{D}$. degree from the University of Western Australia (UWA), Perth, WA, Australia, in 1979.

He was a Research Scientist with Lehigh University, Bethlehem, PA, USA, from 1979 to 1980, where he was involved in studies on MOS devices. From 1980 to 1986, he was a Member of the Technical Staff with RCA Laboratories, David Sarnoff Research Center, Princeton, NJ, USA, where he was involved in very large scale integration (VLSI) CMOS and non-volatile memory technologies, and space radiation effects in siliconon-sapphire MOS integrated circuits. He joined the School of Electrical, Electronic and Computer Engineering, UWA, in 1987, where he has been a Professor since 1998, and the Head of the Department/School from 1999 to 2003. Since joining UWA, his research interests have been in the areas of compound semiconductor materials and devices, and microelectromechanical systems (MEMS). He has supervised more than $30 \mathrm{Ph} . \mathrm{D}$. student completions, and published more than 300 refereed technical papers in journals and conference proceedings.

Prof. Faraone was awarded the RCA Laboratories Individual Outstanding Achievement Award in 1983 and 1986, and the John de Laeter Innovation Award in 1997. He is a Member of the Order of Australia (AM), and a Fellow of the Australian Academy of Science (FAA), the Australian Academy of Technological Sciences and Engineering (FTSE) and the Institute of Electrical and Electronics Engineers (FIEEE). 